\title{
Estimating the parameters of the seabed using the spatial characteristics of ocean ambient noise
}

\author{
He $\mathrm{Li}^{1,2}$, Xinyi Guo ${ }^{1, *}$, Li Ma ${ }^{1}$, and Guoli Song ${ }^{1}$ \\ ${ }^{1}$ Key Laboratory of Underwater Acoustic Environment, Institute of Acoustics Chinese Academy of Science, 100190, Beijing, China \\ ${ }^{2}$ University of Chinese Academy of Science. 100049 Beijing, China
}

\begin{abstract}
When solving traditional underwater problems, the boundary condition is always used to calculate the sound field. In practice, however, it is hard to get the boundary conditions of the seabed. So geoacoustics inversion is needed to acquire the parameters of the seabed. In this paper, a method estimating seabed parameters by using the spatial characteristics of ocean ambient noise is demonstrated without using matched-field processing. For the reason of the limit of the resolution of conventional beamforming (CBF), a method of synthetic array processing (SAP) is used because of some characters of cross-spectrum density matrix (CSDM). The result shows that the method of synthetic array processing enhanced the resolution of critical angle to some degree. By comparing the true bottomloss calculated by OASR, the result of traditional beamforming and the synthetic array processing, the result of synthetic array processing is closer to the true bottomloss than the result of traditional beamforming. After ensuring a range of critical angle, the sound speed of the seabed can be estimated by using Snell law. And then, an experimental data collected in Qingdao, China, 2016 is used to prove the validity of the method of synthetic array processing and estimate the local seabed parameters.
\end{abstract}

\section{Introduction}

In the ocean, the field of ambient noise always exists. In most situations, the field of ambient noise in the ocean which is caused by stormy waves, rain, boat sailing, sea creatures and other industrial activity of human is regarded as an interference of the SONAR system. To some extent, it depends the operating distance of SONAR. Scientists over the world began to study on ocean ambient noise modelling [1-4]. In this paper, the model developed by Kuperman and Ingenito (K/I) will be used and the details will be discussed later [5].

Besides, scientists realized that the characteristic of the field of ocean ambient noise in different sea area is always different. That means the ambient noise field contains several kinds of information in the ocean, such as seabed, the body of water and sea surface. The famous curve of ambient noise spectra developed by Gordon M.Wenz showed that different sources have different characteristics on the spectra [6]. In traditional geoacoustics inversion, active signal is always needed in the practice experiments. However, geoacoustics inversion using ambient noise has much superiority. On the one hand, it doesn't need complicated equipment and sources only a line of array. On the other hand of environment, passive acoustic inversion has no influence on any sea creatures.

In recent years, geoacoustic inversion aroused a great heat. In 2002, Harrison developed a simple method of geoacousic inversion by using ocean ambient noise, that suggests that power reflection coefficient which is a function of angle and frequency is a simple ratio [7]. On this base, Martin Siderius developed a method of synthetic array processing to estimate higher resolution seabed bottom loss [8-9]. Later, Lanfranco Muzi developed a method of frequency based noise coherence-function extension to passively estimate bottom loss [10-11]. In this paper, the method of synthetic array processing is referenced, and estimates the seabed parameters by using Snell law. In this way, the sound speed of the seabed is estimated. As for the density, it may be estimated because of the relationship between density and sound speed [12]. Besides, other information can also be extracted from ocean ambient noise, such as Green's function [13-14]. Also, by using ambient noise, subbottom profiling can be implemented [15-16].

The remainder of this paper is organized as follows. Section 2 summarized the technique to obtain the bottomloss estimates from ambient noise beamforming by Harrison and Simons, and seabed sound speed estimated by Snell law. Section 3 presents the method of synthetic array processing and simulating calculation. After that the results of the technique on experimental data collected in Qingdao, China is shown. And the main findings of this study will be summarized in Section 4.

\footnotetext{
*Corresponding author: guoxinyi@mail.ioa.ac.cn
} 


\section{Passive bottomloss and seabed sound speed estimation}

Harrison and Simons introduced a method of passive bottomloss estimation first [7]. Several studies have proved that it is effective. In the study, the main noise source is regarded as breaking waves and rain distributed uniformly on the sea surface. The ambient noise is sampled by a vertical line array, as shown in fig.1. The noise in the water has been divided into upgoing wave and downgoing wave. After a series of simple derivation, the bottom power reflection coefficient $\mathrm{R}$ is defined as the ratio of the upgoing wave and the downgoing wave. In practice, after beamforming by the vertical line array, the wave from positive receive angle is regarded as downgoing wave, while the wave from negative receive angle is regarded as upgoing wave. This is because the source on the surface is assumed uniformly, and the wave from negative receive angle has been interacted by the seabed, especially in shallow water which has a constant sound speed in the water, so the surface angle and the bottom angle are all equal to the receive angle. In this paper, only water with constant sound speed is taken into account.

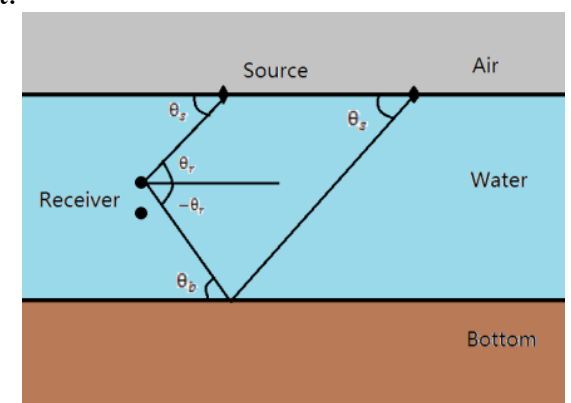

Fig. 1. In shallow water, the surface angle and the bottom angle are all equal to the receive angle because of the constant sound speed. And the power reflection coefficient at $\theta_{r}$ is computed from the signal from the two solid line.

The bottomloss is a function of receive angle, $\theta_{r}$, and frequency, $\omega$, which is defined as

$$
B L\left(\theta_{r}, \omega\right)=-10 \log _{10} R\left(\theta_{r}, \omega\right)
$$

where $R$ is the plane-wave power reflection coefficient of the bottom mentioned previously. As Harrison and Simons shows that the power reflection coefficient can be estimated by the ratio of the upgoing wave and the downgoing wave which, in fact, computed by the array data

$$
R\left(\theta_{r}, \omega\right)=\frac{B\left(-\theta_{r}, \omega\right)}{B\left(\theta_{r}, \omega\right)}
$$

here, $B$ is referred to average beam power, defined as

$$
B\left(\theta_{r}, \omega\right)=w^{H} C_{\omega} w
$$

where $w$ is the weight vector, $w\left(\theta_{r}, \omega\right)=\left[w_{1}, \cdots, w_{M}\right]^{\mathrm{T}}$ ( $\mathrm{T}$ represents the transpose operation). $\mathrm{M}$ is the number of the array elements.

$$
C_{w}=E\left(p p^{H}\right)
$$

$C_{w}$ is the cross-spectral density matrix(CSDM), and E denotes expectation. In Eq. (3) and (4), H represents the conjugate transpose operation. In addition, the vector $p(\omega)$ is the frequency domain signal Fourier transformed from the time domain signal $p(t)$ sampled by $\mathrm{M}$ elements array, which is a matrix with $\mathrm{M}$ lines.

As Eq. (1) shows that BL is a function of $\theta_{r}$ and $\omega$, thus at a particular frequency there is a curve of $B L-\theta_{r}$. In this curve, with the increasing of $\theta_{r}$ from $0^{\circ} B L$ will also increase, especially during the critical angle. Dramatically increase and oscillation will occur when $\theta_{r}$ increase to the critical angle. The simulation will be shown in next section. So a range of critical angle can be confirmed, by using Snell law

$$
c_{1} \cos \theta_{0}=c_{2} \cos \theta_{2}
$$

the sound speed of the seabed can be estimated, where $\theta_{0}$ is the critical angle, $c_{1}$ is the sound speed in the water that is easily acquire, $\theta_{2}$ here is regarded as 0 .

\section{Synthetic array processing and simulation}

\subsection{Synthetic array processing (SAP)}

In conventional beamforming (CBF), the weight vector for the Mth element of the array is

$$
w_{M}=e^{-i(m-1) \frac{w}{c} d \sin \theta_{r}}
$$

and $C_{w}$ is a $\mathrm{M} \times \mathrm{M}$ CSDM. For simplicity, just assume a array with three hydrophones, the CSDM, $C$ (short for $C_{w}$ at particular frequency $\omega$ ), becomes

$$
C=\left(\begin{array}{lll}
c_{1,1} & c_{1,2} & c_{1,3} \\
c_{2,1} & c_{2,2} & c_{2,3} \\
c_{3,1} & c_{3,2} & c_{3,3}
\end{array}\right)
$$

here, $c_{1,2}$ denotes the coherence between hydrophone 1 and 2 , and so on for the CSDM. Because the source is distributed uniformly on the sea surface, the noise in the water is a wide stationary random process. The coherence between two hydrophones is determined by the distance of two hydrophones rather than the absolute depth. $c_{1,2}$ and $c_{2,1}$ are conjugate-symmetric, that is

$$
c(-\mathrm{d})=c^{*}(d)
$$

So Eq. (7) becomes

$$
\begin{aligned}
C= & \left(\begin{array}{lll}
c_{1,1} & c_{1,2} & c_{1,3} \\
c_{2,1} & c_{2,2} & c_{2,3} \\
c_{3,1} & c_{3,2} & c_{3,3}
\end{array}\right) \sim\left(\begin{array}{lll}
c_{1,1} & c_{1,2} & c_{1,3} \\
c_{1,2}^{*} & c_{2,2} & c_{2,3} \\
c_{1,3}^{*} & c_{2,3}^{*} & c_{3,3}
\end{array}\right) \\
& \sim\left(\begin{array}{lll}
c_{1,1} & c_{1,2} & c_{1,3} \\
c_{1,2}^{*} & c_{1,1} & c_{1,2} \\
c_{1,3}^{*} & c_{1,2}^{*} & c_{1,1}
\end{array}\right)
\end{aligned}
$$

Under this circumstance, a synthetic element is assumed above the No.1 hydrophone which is uniformly-spaced, the coherence between this synthetic hydrophone and the true No.1 hydrophone can be presented by the coherence between the true No.1 hydrophone and the true No.2, that is $c_{s, 1}=c_{1,2}^{*}$, where $c_{s, 1}$ is the coherence of the synthetic element and the true No.1. Thus the $3 \times 3 \mathrm{CSDM}$ can be extended into a $5 \times 5$ matrix with the unknown elements in the matrix 
regarded as 0 . Hence the CSDM becomes

$$
C=\left(\begin{array}{ccccc}
c_{1,1} & c_{1,2} & \multicolumn{1}{c}{c_{1,3}} & 0 & 0 \\
c_{1,2}^{*} & c_{1,1} & c_{1,2} & c_{1,3} & 0 \\
c_{1,3}^{*} & c_{1,2}^{*} & c_{1,1} & c_{1,2} & c_{1,3} \\
0 & c_{1,3}^{*} & c_{1,2}^{*} & c_{1,1} & c_{1,2} \\
0 & 0 & c_{1,3}^{*} & c_{1,2}^{*} & c_{1,1}
\end{array}\right)
$$

For the physical interpretation is shown in Fig.2. In a word, this method extends the matrix from $\mathrm{M} \times \mathrm{M}$ to $(2 \mathrm{M}-1) \times(2 \mathrm{M}-1)$.

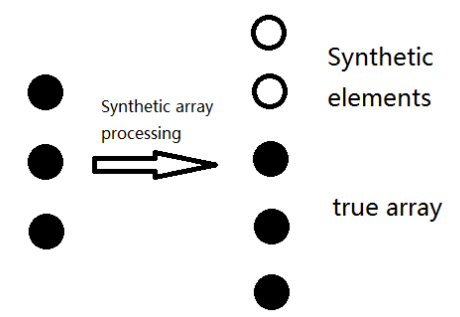

Fig. 2. The physical interpretation of synthetic array processing.

\subsection{Simulation results}

In this section, simulation results will be shown. The noise cross-coherence spectral density matrix is calculated under K/I model as mentioned before. In this model, the problem under horizontally stratified environment has been solved [5]. Kuperman and Ingenito deduced the expression of cross-spectral density function of the noise field as following

$$
\begin{aligned}
& C_{w}\left(R, z_{1}, z_{2}\right)= \\
& \frac{4 \pi^{2} q^{2}}{k^{2}} \int_{-\infty}^{+\infty} g\left(k_{r}, z_{1}, z^{\prime}\right) g^{*}\left(k_{r}, z_{2}, z^{\prime}\right) \times H_{0}^{(1)}\left(k_{r} R\right) k_{r} d k_{r}
\end{aligned}
$$

where $g$ denotes the Green's function. So the CSDM can be calculated with Eq. (11) by using scooter. The parameters are shown in table 1.

Table 1. Parameters for simulation.

\begin{tabular}{|c|c|c|c|c|}
\hline & $c(\mathrm{~m} / \mathrm{s})$ & $\rho\left(\mathrm{kg} / d^{3}\right)$ & $d(\mathrm{~m})$ & $\alpha(\mathrm{dB} / \lambda)$ \\
\hline Water & 1500 & 1 & 30 & 0 \\
\hline $\mathrm{L} 1$ & 1575 & 1.5 & 3 & 0.2 \\
\hline Half space & 1800 & 2 & $\infty$ & 0.6 \\
\hline
\end{tabular}

here $c$ represents the sound speed, $\rho$ is the density of each media, $\mathrm{d}$ is the depth of each layer, $\alpha$ is the attenuation coefficient. The distance between each element is $0.16 \mathrm{~m}$.

Fig.3-6 is the bottomloss computed by different methods. Fig. 3 is estimated by conventional beamforming with true 24 elements. Fig. 4 is calculated by the method of synthetic array processing which extends 24 elements to 47 elements. Fig. 5 is true 47 elements results. As for fig. 6 is the true bottomloss calculated by OASR, the reflection coefficient module of the OASES [17].

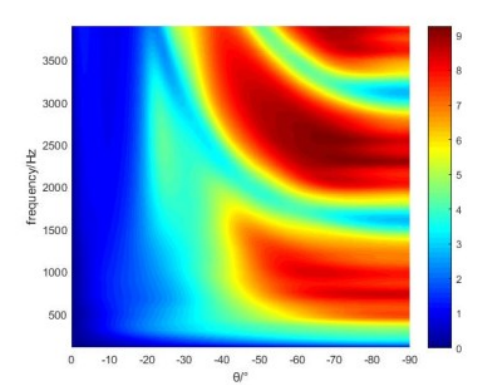

Fig. 3. $B L$ estimated by 24 elements.

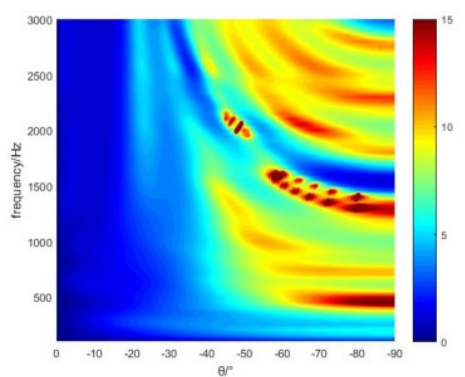

Fig. 4. $B L$ estimated by synthetic array processing extends 24 elements to 47.

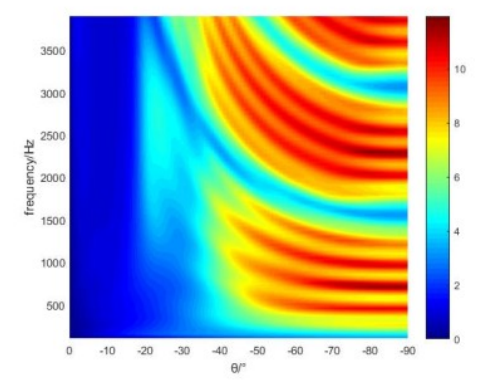

Fig. 5. True 47 elements $B L$.

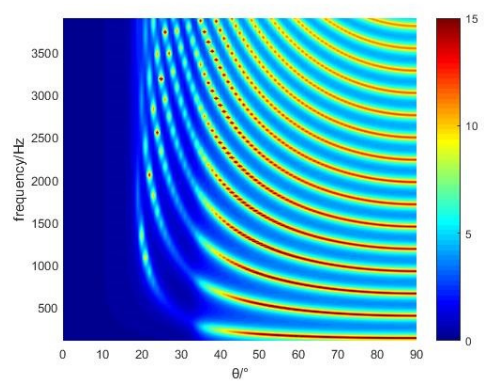

Fig. 6. True $B L$ calculated by OASR.

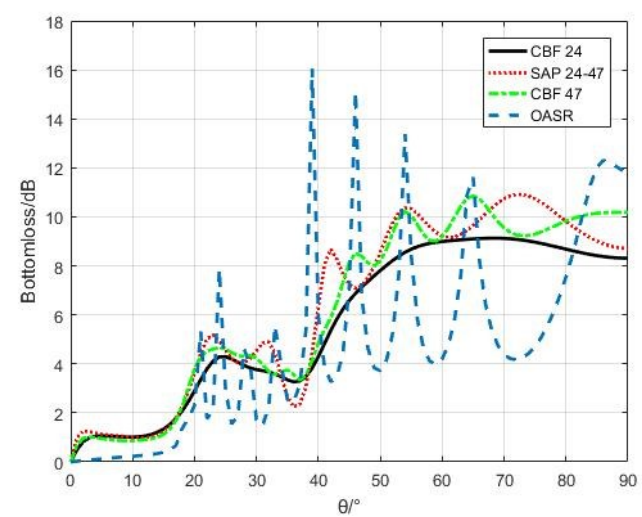

Fig. 7. BL calculated by 4 different methods at $2500 \mathrm{~Hz}$.

In Fig.7, the curve at $2500 \mathrm{~Hz}$ is extracted. The 
results show that the method of SAP's result is much closer to the result of true 47 elements' CBF and the true OASR $B L$ than the 24 elements CBF. And the resolution of the critical angle is more obvious, which is between $18^{\circ}$ to $20^{\circ}$. Then the sound speed of the seabed can be estimated by Snell law. The reason for choosing the frequency of $2500 \mathrm{~Hz}$ is to prevent the interference of low frequency from shipping or other human's activity. Besides the component of high frequency has a poor penetrability that can truly show the characteristics of the seabed.

\subsection{Application of experimental data}

The experimental data is collected in Qingdao, China in 2016 winter. The local water sound speed of the experiment is $1487 \mathrm{~m} / \mathrm{s}$ with the depth of $30 \mathrm{~m}$. The length of the data is 20 minutes and averaged every 2 seconds. There are 25 elements spaced $0.2 \mathrm{~m}$ used in this experiment. $\mathrm{CBF}$ method is used for the last 13 elements and the whole 25 elements. And SAP method is used for the last 13 elements to prove that the method of SAP is valid and estimate the parameters of the seabed. Fig. 8 shows three curves of each method at the frequency of $2600 \mathrm{~Hz}$.

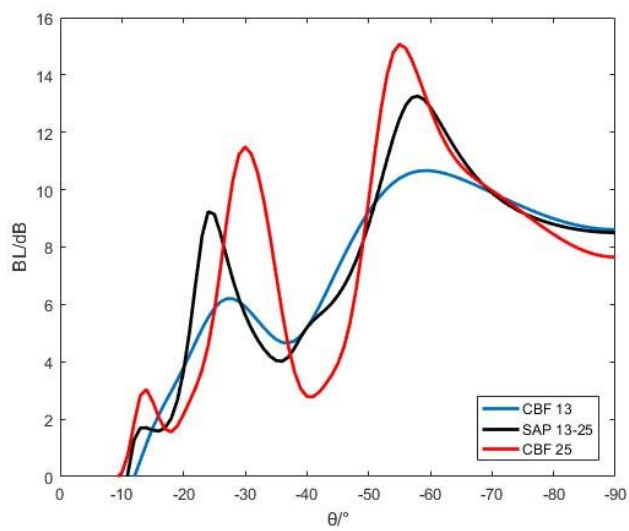

Fig.8. Experimental data $B L$ estimated by three methods at $2600 \mathrm{~Hz}$.

The result indicates that the method of SAP gives a better curve and much closer to the true 25 elements CBF. To some extent, it improves the resolution of the critical angle, which is between $20^{\circ}-25^{\circ}$ probably. So the range of the sound speed of the seabed is roughly from $1600-1650 \mathrm{~m} / \mathrm{s}$. Thus the seabed may mostly consist of sand.

\section{Conclusions}

A method of estimating the parameters of the seabed in constant sound speed shallow water is introduced previously. Because of the limit of the conventional beamforming, a method of synthetic array processing is adopted because of some characteristics of the CSDM that makes the matrix extended from $\mathrm{M} \times \mathrm{M}$ to $(2 \mathrm{M}-1)$ $\times(2 \mathrm{M}-1)$. After simulating calculation and experiment verification, the SAP method improves the resolution of the BL- $\theta$ curve, and by estimating the range of critical angle the sound speed can be estimated using Snell law.

\section{References}

1. B.F.Cron, C.H.Sherman, J. Acoust. Soc. Am. 34, 1732-1736(1962)

2. C.H.Harrison, J.Acoust.Soc.Am. 99,2055-2066 (1996)

3. C.H.Harrison. Applied Acoustics. 51,289-315 (1997)

4. Michael J.Buckingham, J.Acoust.Soc.Am.134, 950-958(2013)

5. W.A.Kuperman, F.Ingenito, J.Acoust.Soc.Am.67, 1988-1996(1980)

6. Gordon M.Wenz, J.Acoust.Soc.Am. 34,19361956(1962)

7. C. H. Harrison, D. G. Simons, J. Acoust. Soc. Am. $112,1377-1389(2002)$

8. Martin Siderius, Lanfranco Muzi, Chris $H$. Harrison, Peter L. Nielsen, Acoust. Soc. Am. 133, 149-155 (2012)

9. Martin Siderius, Chris Harrison. AIP Conf. Proc. 728, 22-31 (2004)

10. Lanfranco Muzi, Martin Siderius, Jorge E. Quijano, Stan E. Dosso, J. Acoust. Soc. Am. 137, 481-491 (2014)

11. Lanfranco Muzi, Martin Siderius, Peter L. Nielsen, J. Acoust. Soc. Am. 140, 1513-1524 (2016)

12. Finn B. Jensen, William A. Kuperman, Michael B. Porter, Henrik Schmidt, Computational Ocean Acoustics, 2nd Edition. Springer, New York, 2011. Chapter 1.6, P39

13. S. E. Fried, W. A. Kuperman, Karim G. Sabra, Philippe Roux, J. Acoust. Soc. Am. 124, 183- 188 (2008)

14. Karim G. Sabra, Peter Gerstoft, Philippe Roux, W. A. Kuperman, Michael C. Fehler, Geophysical research letters, 32, L03310 (2005)

15. Chris H. Harrison, J. Acoust. Soc. Am. 115, 15051515 (2003)

16. Martin Siderius, Chris H. Harrison, Michael B. Poter, J. Acoust. Soc. Am. 120, 1315-1323 (2006)

17. H. Schmidt, OASES Version 3.1, User Guide and Reference Manual (Massachusetts Institute of Technology, Cambridge, MA, 2004) 